\title{
Effects of antecedent order and semantic context on Chinese pronoun resolution
}

\author{
HSUAN-CHIH CHEN \\ Chinese University of Hong Kong, Hong Kong \\ HIM CHEUNG \\ University of Otago, Dunedin, New Zealand \\ and \\ SIU LAM TANG and YUK TAK WONG \\ Chinese University of Hong Kong, Hong Kong
}

\begin{abstract}
Three reading time experiments were conducted in order to examine the relative contributions of order of antecedents and semantic context to the resolution of temporarily ambiguous Chinese pronouns. These pronouns were ambiguous because each of them was preceded by two antecedents, both of which were likely candidates for coreference. The identity of the pronoun was revealed by subsequent disambiguating information that constrained the pronoun to one particular interpretation. Experiment 1 showed that reading of the disambiguating phrase was slower when the phrase confined the pronoun to the second rather than to the first antecedent. Experiment 2 produced the same effect of antecedent order (first vs. second antecedent) regardless of whether the target antecedent was an actionperforming or an action-receiving entity. In Experiment 3, the order effect was eliminated by a biasing modifier inserted immediately before the pronoun. These results indicate that in a semantically neutral environment, the first-appearing antecedent is the preferred candidate for coreferencing the ambiguous Chinese pronoun. The interaction between order of antecedents and semantic context (in the form of preposed biasing modifiers) suggests that the initial comprehension of Chinese pronouns depends as much on contextual as on structural factors.
\end{abstract}

\section{Consider the following sentence:}

I saw John and Mike sitting on the pier; he was watching the sea.

The identity of the ambiguous pronoun "he" is undefined, since both the antecedents, "John" and "Mike," are probable candidates for coreference. In this situation, at least two possibilities exist regarding how the reader might resolve the pronoun. First, perhaps one unique antecedent is immediately identified as the coreferent, despite the lack of linguistic constraints. For instance, the reader might assign the pronoun immediately upon reading it to "John" simply because "John" is the first antecedent. Alternatively, both "John" and "Mike" might be equally activated initially, and selection of the more probable candidate might not begin until a later stage, when more contextual information becomes available. The former view emphasizes immediate pronoun processing on the basis of struc-

This research was supported by Earmarked Grants from the Research Grants Council of Hong Kong to H.-C.C. We thank Angela Wong and Jackie Tsang for their extensive assistance during various phases of these experiments. The manuscript benefited from comments and suggestions by M. A. Gernsbacher and two anonymous reviewers. Correspondence concerning this article should be addressed to H.-C. Chen, Department of Psychology, Chinese University of Hong Kong, Shatin, N.T., Hong Kong (e-mail: hcchen@psy.cuhk.edu.hk). tural (syntactic) cues that are readily available, and the cue in this example is the relative order of the antecedents. The latter view attends to the role played by contextual factors, the effect of which is, in this case, delayed.

Crawley, Stevenson, and Kleinman (1990) obtained evidence for a syntactically based pronoun-processing strategy that is biased toward assigning the pronoun to the antecedent occupying the grammatical subject position. The authors had their subjects read passages, each of which contained an ambiguous pronoun preceded by two antecedents-for example,

Sarah visited Cathy at home and Charles rang her at work.

In the assignment task, subjects decided explicitly whether the pronoun referred to the first (subject) or the second (object) character. It was found that readers interpreted the pronoun more frequently as the subject than as the object antecedent, although the pronoun itself was always a grammatical object. Subject-antecedent interpretations were also made faster than object-antecedent interpretations. Furthermore, averaged per-word reading time for the critical sentence was shorter when the pronoun was understood as the subject than when it was understood as the object antecedent. These results were taken as evidence of an assignment bias toward the antecedent occupying the subject position when there was a lack of constraining information. 
Another way to make inferences about pronoun resolution is to present the antecedents in isolation as probes for yes-no recognition after sentence reading. Differential recognition speeds and accuracies are supposed to indicate different levels of activation owing to the antecedents' (probes') differential associations with the pronoun. The identified coreferent is closely associated with the pronoun, and thus, the reading of the pronoun would reactivate its representation. This facilitates recognition as measured by both speed and accuracy, when the coreferent is later presented as a probe, as compared with the unidentified antecedent. The sensitivity of this method was demonstrated in an early study by Chang (1980), who presented two-clause sentences in which the first clause contained a critical and a noncritical proper name (e.g., "John and Mary went to the grocery store ..." with "John" being the critical name, because it would reappear as the test probe). Recognition of the critical name probe after reading the whole sentence was faster when the second clause contained a gender-appropriate pronoun (i.e., the critical pronoun; e.g., "... and he bought a quart of milk") than when it contained a pronoun that could only refer to the noncritical name (i.e., the control pronoun; e.g., "... and she bought a quart of milk"). Such a facilitatory effect was specific to the recognition of the critical name probe, thus pointing to the notion that reading of a pronoun reactivates only the semantic representation of its unique coreferent. Using this method, Corbett and Chang (1983) demonstrated that the processing of ambiguous pronouns did not lead to immediate identification of unique coreferents. The resolution of temporarily ambiguous pronouns might be delayed, pending useful contextual cues. The authors had their subjects read two-clause sentences, each of which contained two proper names and an ambiguous pronoun (or a proper name) in its first and second clauses, respectively-for example,

Gary was interviewed by Marvin but (Gary/he) would not answer many questions.

Subjects made a yes-no recognition response to a probe, which could be the actual coreferent or the noncoreferent, presented immediately after the test sentence. The authors reported faster probe recognition times and higher recognition accuracies for noncoreferent probes when the second clause contained a pronoun than when it contained a proper name. This result supported the hypothesis of an initial activation even of the noncoreferent when the reader later came across a pronoun, since facilitation in recognition speed and accuracy for the noncoreferent was not observed when a proper name, rather than a pronoun, was used in the second clause.

Nevertheless, because Corbett and Chang (1983) had their subjects respond to the test probe only after reading the whole sentence, there was no way to determine whether activation of the coreferent and the noncoreferent, as indicated by facilitation in probe verification, was indeed due to pronoun processing. The antecedents might have already been activated to that level prior to the pronoun. The lack of facilitation in the proper name control condition could indicate that activation simply dissipated faster in this condition. To test this hypothesis, Gernsbacher (1989) placed a test point both immediately before and immediately after the anaphor (i.e., a repeated proper name or a pronoun). If activation of the antecedents was due to pronoun processing, facilitation in probe verification should be greater for those subjects responding after the anaphor than for those responding before the anaphor. The results, however, showed that facilitation was comparable in the two conditions. This contradicts the notion that reading a pronoun activates its coreferent as well as other previously appearing noncoreferents. These entities are activated simply because they have just been processed in a recent clause. One further finding was that, prior to the anaphor, the first-mentioned antecedent tended to be more activated than the second-mentioned antecedent. This effect of first mention dissipated faster with repeated proper name anaphors than with pronoun anaphors. Hence, on the basis of the probe verification data, there is no clear evidence that unselective activation of all possible antecedents is triggered by pronoun processing. A more general mechanism, which gives rise to the effect of first mention, appears to be responsible for differentially activating the antecedents.

The question of whether reading a pronoun would activate only its coreferent was further investigated in a series of nine experiments by Greene, McKoon, and Ratcliff (1992), who varied the positions of test words (i.e., probes) within texts, so that sometimes they were presented before and sometimes after the critical pronouns. The test words were either coreferents or noncoreferents of the critical pronouns. If the reading of a pronoun activated only its coreferent, different levels of probe recognition performance associated with different probe positions would be expected only for coreferent probes, not for noncoreferent probes. Greene et al. did not establish the expected interaction in their first four experiments, thus calling into question the notion of early coreferent identification. However, the authors showed that if the task encouraged the identification of coreferents, a recognition advantage of coreferent over noncoreferent probes after pronoun reading could actually be generated. It was reported in Experiments 5, 6, and 7 that task demand and a slow rate of presentation led to unique identifications. The authors thus concluded that the pronoun resolution process is nonautomatic and sensitive to contextual factors.

Some recent studies have used text reading times to reveal how temporarily ambiguous pronouns are processed. If the ambiguous pronoun were initially assigned to a unique antecedent, text reading would be slowed if the reader later encountered information that contradicted the initial assignment. This method was used by Gordon and Scearce (1995), who demonstrated an interplay between initial assignment bias and semantic context. The participant read sentences containing temporarily ambiguous pronouns, the identities of which did not become clear 
until reading came to a disambiguation region. The disambiguation region (italicized in the quotation below) supplied information that semantically confined the assignment to one of the two antecedents - for example,

Bill wanted John to look over some important papers. He had to mail him the documents by Monday. Unfortunately, he never sent/received the papers.

It was found that reading time for the disambiguation region was significantly longer when the disambiguating information contradicted than when it confirmed a subject-antecedent interpretation of the pronoun. This effect, nevertheless, disappeared when proper names, rather than pronouns, were used. This result suggests an automatic pronoun resolution mechanism that biases assignment toward the previous subject noun phrase, echoing the conclusion drawn by Crawley et al. (1990). In another experiment, Gordon and Scearce (1995) examined the role played by semantic context and investigated whether the early provision of confining information would block the automatic assignment mechanism. Semantically disambiguating information (italicized in the quotation below) was now presented before the ambiguous pronoun-for example,

Bill wanted John to look over some important papers. He had to mail him the documents by Monday. After sending/receiving the papers, he began more work.

If the automatic subject assignment tendency persisted despite the preposed information (i.e., the context), a pattern of results similar to that of the first experiment would be observed with respect to reading times in the region set in boldface. As was expected, the main finding of the first experiment was replicated, although the magnitude of the effect was reduced by the preposed information. Taken together, these findings are indicative of a robust structural effect (i.e., a subject-antecedent bias) that interacts with the semantic environment. Whether unique coreferents are automatically identified for pronouns, therefore, appears to be a matter of degree.

Research on pronoun resolution has so far focused only on the English language. Thus, the contention that the subject-antecedent assignment bias constitutes a universal mechanism that generalizes to other natural languages would need cross-language verification. English, as compared with most Asian languages, is structurally transparent, in that there is a relatively regular correspondence between syntactic constructions on the surface and meaning at a deeper level. Grammatical devices override semantic context and guide comprehension in an explicit fashion. This general emphasis on syntactic processing may have contributed to the language user's sensitivity to structural cues when encountering ambiguous pronouns. Very different assignment strategies may be used in languages that rely less on syntax but more on contextual information for comprehension.

Chinese, a widely used Asian language, is characterized by its lack of inflections and well-defined grammatical devices. This foregrounds the importance of semantic context and other nonsyntactic cues in comprehension (Chen, 1992, 1996; C. Li \& Thompson, 1981). When we call Chinese a context-dependent language, we are referring to the general fact that, in this language, the comprehension of low-level elements is heavily influenced by the interpretation of high-level structures. For example, a typical modern Chinese word is composed of two characters, which are lexical morphemes, each being associated with a few alternative meanings. Which meaning a character (low-level element) should take on is determined by the word (high-level structure) comprising it. This does not happen with an English morpheme, whose meaning is typically invariant across different "host" words. Another example has to do with the interpretation of Chinese verbs. Since Chinese verbs do not inflect, their aspectual/ temporal characteristics are revealed either through the immediate context, in the form of aspect markers, or via the more remote phrasal/sentential environment. In other words, the comprehension of Chinese characters/words involves a diffused, semantically oriented strategy, whereas deciphering English words is a more focused, structuredependent activity. This argument is empirically grounded. For instance, Chen (1992) reported that reading times for Chinese characters were better predicted by sentence-level variables, such as number of new argument nouns and syntactic boundaries in the sentence, than by characteror word-level variables. This contrasts with the standard English finding that word-level variables are the most robust predictors of word reading times (e.g., Haberlandt \& Graesser, 1985; Just \& Carpenter, 1980). Furthermore, a few studies have suggested the dominant use of semantic cues in the Chinese reader's assignment of theta roles (P. Li, Bates, Liu, \& MacWhinney, 1992; Miao, 1981). In this context, a theta role indicates how a noun phrase relates to the main verb. For example, in the sentence "John hit Mike," the noun phrase "John" takes on an agent role, since it refers to an entity performing the "hit" action. "Mike" has the theta role of patient, because it represents an entity receiving the action. In English, the assignment of theta roles is typically determined by word order (see MacWhinney, 1987). The Chinese reader's reliance on semantic cues constitutes another example of context dependency in Chinese comprehension.

Some previous Chinese studies have used grammatically implausible sentences that could have forced the reader to back off from syntactic analyses and adopt a semantic strategy when performing comprehension tasks (e.g., P. Li et al., 1992; Miao, 1981). Thus, further studies, using more natural test material, are needed for a fair test of the effects of both structural and semantic variables. The present study accomplishes this goal by investigating pronoun resolution in Chinese, using grammatically and stylistically natural passages in a moving-window paradigm. It evaluates how the structural factor of order of antecedents interacts with semantic context in Chinese pronoun resolution. Three reading time experiments were reported. In Experiment 1, the effect of antecedent 
order was examined in a situation in which the two antecedents carried the same theta role. Experiment 2 contrasted the orthogonal effect of antecedent order with that of theta role. Experiment 3 tested the effect of semantic context over and above the antecedent order effect. These experiments adopted the moving-window technique to record character-by-character reading times of Chinese passages, each of which was divided into four regions. The first region extended from the beginning of the passage to the point just before the ambiguous pronoun. This region contained the two antecedents. The second, or the ambiguity, region stretched from the ambiguous pronoun to the point just before the disambiguating information. The third, or the disambiguation, region extended from the beginning to the end of the disambiguating phrase. The reader should have developed a fairly clear idea about the identity of the pronoun by the end of this region. The final region covered the rest of the passage. The general logic of the present experiments was that if information in the third region disambiguated the pronoun as being the same antecedent as the one that was also suggested by the hypothetical assignment mechanism to be the coreferent, reading times in the disambiguation region would be shorter than they would be in the case in which the disambiguating information ran counter to the hypothetical bias, because reading would be disrupted in the latter but not in the former situation. By manipulating the disambiguating information and examining the pattern of third-region reading times, inferences about the initial assignment bias could be made.

\section{EXPERIMENT 1}

Some past research has shown that an ambiguous pronoun is more frequently interpreted as coreferring to the subject than to the object in a previous clause (Chen \& Hung, 1995; Crawley et al., 1990; Gordon \& Chan, 1995; Gordon, Grosz, \& Gilliom, 1993; Matthews \& Chodorow, 1988). Nevertheless, these studies did not separate the effect of the subject/object status from the effect of the order of appearance of the antecedents. In the present experiment, the pure effect of appearance order was examined by manipulating information in the third region so that it disambiguated the critical pronoun as either the first- or the second-appearing antecedent. The two antecedents in each passage were equivalent in terms of subject/object status and theta role. They were two parallel, juxtaposed noun phrases connected by a conjunction. The two antecedents equaled each other in all syntactic aspects, except that one preceded the other physically. If antecedent order of mention is important in Chinese pronoun resolution and the assignment bias is toward the first-appearing antecedent, reading in the disambiguation region would be slowed when information in the region disambiguated the critical pronoun as the second antecedent.

Each experimental passage frame had two versions, defining the two conditions of the experiment. The first referencing (first-ref) and the second referencing (secref) versions disambiguated the pronoun as the first and the second antecedents, respectively. Examples of test passages are shown in Figure 1.

\section{Method}

Participants. Twenty-four undergraduates at the Chinese University of Hong Kong participated in the experiment for course credit. They were native Chinese speakers/readers with normal or corrected-to-normal vision.

Apparatus. Stimulus presentation and data collection were controlled by an IBM 486 compatible computer. The stimuli were presented on a multiscan color monitor, using the modified movingwindow method, in which a mechanical mouse was linked to the computer for the control of stimulus presentation (see Chen, 1992, 1999). By moving the mouse from left to right, the participant viewed successive characters of a text, each of which was presented in a spatially appropriate location on the screen. The Chinese characters were input by using the ET Chinese word-processing system and were converted into graphical codes and displayed in graphical format. Each character was composed of illuminated (white) dots and was set against a dark background with a potential $16 \times 16 \mathrm{dot}$ matrix.

Design and Materials. Twelve target passage frames were constructed. Each passage consisted of two grammatically equivalent antecedents and a temporarily ambiguous pronoun. The ambiguous pronoun was subsequently disambiguated in the disambiguation region, which contained descriptive information about the pronoun that identified it as one particular antecedent. The antecedents consisted of both common nouns (58.3\%; e.g., "nurse") and proper names ( $41.7 \%$; e.g., "Cathy"). A third, irrelevant entity (i.e., a noun to which the ambiguous pronoun could not refer) always preceded the first antecedent, so that any observable first-antecedent assignment effect could not be due to the first antecedent's passage-initial position (Gernsbacher \& Hargreaves, 1988). Thirty-four filler passages were also constructed; 10 of them were used in the practice trials. Presentation of target and filler passages was counterbalanced. Each participant read both first-ref and sec-ref passages, yet only one version from each sentence frame was read by any individual participant.

Procedure. The participants were tested individually and were given instructions at the beginning of the experiment. They were told that they would read some Chinese passages and the purpose of the experiment was to investigate the comprehension processes involved in Chinese reading. The task was to read the passages carefully and to answer a comprehension question at the end of each passage.

The onset of each experimental passage was initiated by pressing the space bar on the keyboard. Upon doing so, the screen would be filled with horizontally arranged line segments and punctuation marks. The appearance of characters was controlled by moving the mouse from left to right. The position of the mouse was represented on the screen by a pointer that moved horizontally underneath the line segments; a character corresponding to a certain position was displayed right above the line segment when the pointer came to that position. The appearance of a character above a certain line segment would result in the disappearance of the preceding character or punctuation mark. The exposure duration of a certain character was, therefore, taken and recorded as the reading time for that character. The participant was instructed to set the mouse to the starting position at the beginning of the passage and to proceed by moving it from left to right at his/her own pace. At the end of a line or a page, a right-to-left return sweep was required to move the mouse to the next beginning position. A true-or-false comprehension question was asked after the participant had finished reading each passage. Responses to the comprehension questions were recorded by the experimenter. 


\section{English Translation of a Sample Sentence Frame Used in Experiment 1:}

I was strolling along the seaside when I saw the grandpa and his grandson from my neighborhood sitting on the pier. He was watching the sea as he talked about (his splendid deeds in his youthful times/his school life with his little classmates). They really talked about everything.

\section{First Referencing (First-Ref) Version:}

我 在海尰散步,

I (locative) sea side stroll,

看到 鄰家的爺爺和 孫兒 坐 在 碼頭上, see neighbor (possessive) grandpa and grandson sit (locative) pier on, / 1 他一邉看著海景,

(1) he one side look at sea view,

一遥訴說 182當 年年青時候的風光偉䋖。 one side talk about /(2) that year young time (possessive) splendid deeds.

/(3)他倆 真是 無所 不 談。/

/(3) They really nothing don't talk./

\section{Second Referencing (Sec-Ref) Version:}

我在海透散步,

I (locative) sea side stroll,

看到 搂家 的 爺齐 和 孫兒 坐 在 碼頭上,
see neighbor (possessive) grandpa and grandson sit (locative) pier on, 1他一逼看著海景,

(1) he one side look at sea view,

一透 訴說 1 (2) 在 學校 衰與小同學 的生活。 one side talk about /(2) (locative) school in and little classmates (possessive) life. /3他倆 真是無所 不 談。/

/(3) They really nothing don't talk./

$$
\begin{aligned}
& \text { (1) _ ambiguity region } \\
& \text { /(2) - disambiguation region } \\
& \text { (3) - final region }
\end{aligned}
$$

Figure 1. Sample passages used in Experiment 1 and their English translations.

\section{Results and Discussion}

Effectiveness of manipulation. Ten independent undergraduates at the Chinese University of Hong Kong decided on the identity of the pronoun for each target passage. In addition, 12 neutral passages were constructed and used as controls. The third (disambiguation) region of these neutral passages contained information that ap- plied to both antecedents- that is, the third region did not disambiguate the pronoun. Self-ratings on how confidently pronoun assignments were correctly made were also obtained with a 5-point scale $(1=$ very unconfident, $5=v e r y$ confident ). Contrasting the first-ref and sec-ref target passages with the neutral passages on both the actual pattern and the confidence of pronoun assignment 
would thus reveal how biasing, or effective, the disambiguating information was. Results indicated that, in 96.7\% of the first-ref passages, the first antecedent was considered the coreferent, whereas in $90.8 \%$ of the sec-ref passages, the second antecedent was taken as the coreferent. For the neutral passages, the figures for first- and secondantecedent assignment were $79.2 \%$ and $20.8 \%$, respectively. It was obvious that the disambiguating information in the first-ref and sec-ref passages was indeed biasing toward the expected directions, as compared with the neutral condition, in which first- versus second-antecedent assignment was less asymmetric. This was further confirmed by the confidence scores: First-antecedent assignment was more confidently done in the first-ref (4.0) than in the neutral passages $[2.8 ; t(9)=8.17, p<.01]$; second-antecedent assignment was more confidently done in the sec-ref (4.0) than in the neutral passages [2.7; $t(9)=7.08, p<.01]$.

The passages were also presented to judges, with the disambiguating phrases being taken off and not replaced by neutral phrases. The judges' decisions on what the pronoun referred to therefore reflected how ambiguous the original passage frame was. A second version was created for each original frame so that the positions of the antecedents were reversed in the new version. Ten independent judges made pronoun assignment decisions for each version. When the antecedents appeared in the original order, $77.7 \%$ of the decisions were first-antecedent assignments (second-antecedent assignments were 22.3\%). Reversing the antecedent positions resulted in $41.7 \%$ and $58.3 \%$ for first- and second-antecedent assignments, respectively.

Comprehension accuracy rates. In the main test, about $93 \%$ and $85 \%$ of the comprehension questions were answered correctly for the filler and the target passages, respectively. ${ }^{1}$ These percentages indicated that the majority of the passages were properly read and comprehended. ${ }^{2}$

Character reading times. Only data from properly understood passages (with correctly answered comprehension questions) were analyzed. Mean character reading times were derived for the ambiguity, disambiguation, and final regions. Mean character reading times were calculated by dividing the total reading time for each region by the number of characters in that region. They are presented in Table 1.

Both by-subject $\left(t_{1}\right)$ and by-item $\left(t_{2}\right) t$ tests were conducted in order to examine differences in character reading times between the first-ref and the sec-ref conditions. The only significant effect was found in the disambiguation region $\left[t_{1}(23)=2.33, p<.05\right]$, but it was only marginal for the by-item analysis $\left[t_{2}(11)=2.06, p=.06\right.$, indicating that mean character reading time for this region was longer in the sec-ref than in the first-ref condition. The main hypothesis of this experiment was supported by the by-subject analysis result, in that further information favoring the second over the first antecedent as the pronoun coreferent slowed reading in the region providing such information. Although the by-item analysis re-
Table 1

Mean Character Reading Times (in Milliseconds; With Standard Deviations) in Experiment 1

\begin{tabular}{|c|c|c|c|c|}
\hline \multirow[b]{3}{*}{ Region } & \multicolumn{4}{|c|}{ Passage Type } \\
\hline & \multicolumn{2}{|c|}{ First Referencing } & \multicolumn{2}{|c|}{ Second Referencing } \\
\hline & $M$ & $S D$ & $M$ & $S D$ \\
\hline Ambiguity & 314.2 & 128.7 & 318.8 & 136.8 \\
\hline Disambiguation & 310.6 & 122.8 & 333.0 & 151.1 \\
\hline Final & 269.2 & 109.1 & 274.2 & 117.0 \\
\hline
\end{tabular}

sult was only marginally significant, it clearly revealed a consistent trend.

\section{EXPERIMENT 2}

Experiment 2 tested the orthogonal effects of antecedent order and theta role of antecedents on the resolution of temporarily ambiguous Chinese pronouns. It disentangled the two effects by using passages consisting of either active or passive constructions, so that the actionperforming entity was represented either by the first (as in active phrases) or the second (as in passive phrases) antecedent. If antecedent order was the more important factor and the pronoun was more likely to be linked to the first antecedent regardless of its theta role, reading of the disambiguating information would be slowed when the information suggested a second-antecedent resolution. If theta role was important and the ambiguous pronoun was always identified with the agent of the action, reading of the disambiguating phrase would be slowed when it suggested the patient of the action as the coreferent. A more complicated pattern of reading times would result if the two factors interacted.

Each experimental passage frame had four versions, defining the four conditions of the experiment. They were (1) the agent and first antecedent (agt-first) version, in which the pronoun was disambiguated as the first antecedent that was also the action-performing agent; (2) the patient and second antecedent (pat-sec) version, in which the pronoun was disambiguated as the second antecedent that was also the action-receiving patient; (3) the agent and second antecedent (agt-sec) version, in which the pronoun was disambiguated as the second antecedent that was also the action-performing agent; and (4) the patient and first antecedent (pat-first) version, in which the pronoun was disambiguated as the first antecedent that was also the action-receiving patient. Versions 1 and 2, and Versions 3 and 4 involved active and passive constructions, respectively. The Chinese passive marker "bei" was used in passive sentences. Examples of test passages are shown in Figure 2.

\section{Method}

Participants. Sixteen undergraduates at the Chinese University of Hong Kong participated for course credit. All the participants were native Chinese speakers/readers with normal or corrected-tonormal vision. 


\section{English Translation of a Sample Sentence Frame Used in Experiment 2:}

Everytime when I see grandpa lift and cuddle Johnny, he always makes (Johnny/grandpa) laugh, and this cheers everybody up.

\section{The Agent and First Antecedent (Agt-First) Version:}

每次當我見到祖父抱起強仔的時候，

Everytime when I see grandpa cuddle lift Johnny (possessive) the time,

/(1) 他總是逗得 / (2) 強仔 / (3) 滿面笑容,

(1) he always make/(2) Johnny/(3) full face laughter,

使 大家 也 高興起來。/
make everybody also happy ./

The Patient and Second Antecedent (Pat-Sec) Version:

每次當, 我見到祖父抱起強仔的時候,

Everytime when I see grandpa cuddle lift Johnny (possessive) the time,

/(1) 他總是逗得 / (3) 祖父 / (3) 满面笑容 ,

(1) he always make/(2) grandpa/(3) full face laughter,

使 大家 也 高興起來。/

The Agent and Second Antecedent (Agt-Sec) Version:

每次當我見到強仔被祖父抱起的時候,

Everytime when I see Johnny (passive marker) grandpa cuddle lift (possessive) the time,

/(1) 他總是逗得 / (2) 強仔 / (3) 満面笑容,

(1) he always make/(2) Johnny/(3) full face laughter,

使大家 也 高興起來。/

make everybody also happy ./

The Patient and First Antecedent (Pat-First) Version:

每次當我見到強仔被祖父抱起的時候,

Everytime when I see Johnny (passive marker) grandpa cuddle lift (possessive) the time,

/(1) 他總是 逗得 / (2) 祖父 / (3) 滿面笑容，

/(1) he always make/(2) grandpa/(3) full face laughter,

使大家也 高興起來。/

make everybody also happy ./

(1)-ambiguity region

(2) - disambiguation region

(3)-final region

Figure 2. Sample passages used in Experiment 2 and their English translations. 


\begin{abstract}
Apparatus. The apparatus was the same as that in Experiment 1 Design and Materials. Twenty new target passage frames were constructed. The basic structure of these passages was similar to that used in Experiment $I$, except that the two antecedents in each passage now took on different theta roles. In these new passage frames, $75.0 \%$ of the antecedents were proper names, whereas the remaining $25.0 \%$ were common nouns. The disambiguating information was available four or five characters after the critical pronoun. Eighty actual target passages resulted from developing four versions for each of the 20 frames. These passages were sorted into four files; each passage frame was represented once and only once in each file. Five target passages from each version were incorporated into each file. A further set of 38 passages was added into each file, serving as fillers. Eight out of the 38 filler passages were used in the practice trials. Presentation of the passages was random, and the same ramdom sequence was used across the four files. Each participant read one file of passages, and each file was read by 4 participants.

Procedure. The procedure was the same as that in Experiment 1.
\end{abstract}

\section{Results and Discussion}

Effectiveness of manipulation. Ten independent undergraduates at the Chinese University of Hong Kong decided on the identity of the pronoun in each target passage. Both active and passive neutral passages were constructed and used as controls. These neutral passages did not contain any biasing (disambiguating) information in the third region. Self-ratings on the reader's confidence in correctly resolving the pronouns were also obtained with a 5 -point scale $(1=$ very unconfident, $5=$ very confident $)$. Contrasting the target with the neutral passages on both the pattern and the confidence of pronoun assignment would thus reveal the effectiveness of the disambiguating information. The results indicated that $97.5 \%$ and $95.5 \%$ of the agt-first and pat-first passages, respectively, received a first-antecedent solution, whereas $95.5 \%$ of both the agt-sec and the pat-sec passages received a secondantecedent solution. For the active neutral passages, a first-antecedent strategy was adopted only $81.5 \%$ of the time; it dropped to $67.5 \%$ for the passive neutral passages. The disambiguating information carried in the four types of target passages was effective in that it biased assignment toward the desired directions. The neutral passages, on the other hand, were associated with a less asymmetric first- versus second-antecedent assignment pattern. This is further confirmed by confidence scores: agt-first (4.6, first-antecedent resolution) versus active neutral [3.4, first-antecedent resolution; $t(9)=4.43, p<.01$ ]; pat-first (4.5, first-antecedent resolution) versus passive neutral [3.3, first-antecedent resolution; $t(9)=3.55, p<$ $.01]$; agt-sec (4.4, second-antecedent resolution) versus passive neutral [3.6, second-antecedent resolution; $t(9)=$ $2.67, p<.05]$; and pat-sec $(4.3$, second-antecedent resolution) versus active neutral $[3.2$, second-antecedent resolution; $t(9)=3.17, p<.05$ ]. The disambiguating information available in the test passages increased the reader's confidence in making pronoun assignment judgments, as compared with the control passages.

The passage frames were also presented to another group of judges without any disambiguating or neutral phrases. The judges' pronoun assignment decisions in this situation would thus indicate how ambiguous the original frames were. A second version was created for each original frame in such a way that the positions of the antecedents were reversed in the new version. Ten independent judges decided on the identity of the pronoun for each version. Results showed that $67.2 \%$ and $62.5 \%$ of the responses were first-antecedent assignment decisions for the original and the reversed versions, respectively.

Comprehension accuracy rates. Overall, about $96 \%$ and $86 \%$ of the comprehension questions were correctly answered for the filler and the target passages, respectively. These percentages indicated that the majority of the test passages were properly read and comprehended.

Character reading times. Only data from properly understood passages (with correctly answered comprehension questions) were analyzed. Mean character reading times were derived for the ambiguity, disambiguation, and final regions. Mean character reading times were calculated by dividing the total reading time for each region by the number of characters in that region. They are presented in Table 2.

Since the theoretically interesting comparisons concern character reading times in the disambiguation region, the following analyses focus on this region only. Both by-subject $\left(F_{1}\right)$ and by-item $\left(F_{2}\right)$ analyses of variance (ANOVAs) were performed, with order (first vs. second antecedent) and theta role (agent vs. patient of the action) as independent factors. The effect of order was significant with the by-subject analysis $\left[F_{1}(1,15)=7.94\right.$, $\left.M S_{\mathrm{e}}=7,168, p<.02\right]$, but not with the by-item analysis $\left(F_{2}<1\right)$. The by-subject analysis result indicated that reading of the disambiguation region was slowed when the information disambiguated the pronoun as the second antecedent, as compared with disambiguating it as the first antecedent. The effect of theta role $\left[F_{1}(1,15)=1.28\right.$, $\left.M S_{\mathrm{e}}=1,994, p>.05 ; F_{2}<1\right]$ and the two-way interaction failed to reach significance (both $F_{1}$ and $F_{2}<1$ ).

The present by-subject analysis results reinforced the main finding of Experiment 1 and supported the hypothesis that ambiguous Chinese pronouns are frequently interpreted as the first-appearing antecedent, regardless of the theta role of that antecedent. If theta role is considered a factor more semantic than structural in nature, its lack of an effect in relation to an observable effect of antecedent order might suggest the importance of syntax in the initial comprehension of a language as contextdependent as Chinese. Nevertheless, it would be unfair to conclude at this point that semantic factors do not play any part in resolving Chinese pronouns, since the theta role manipulation might not have introduced enough variation to be regarded as a true semantic manipulation: Whether an antecedent is the agent or the patient of an action did not carry specific information that explicitly identified the following pronoun. In Experiment 3, we examined in a more direct way the effect of explicit, preposed semantic information on the resolution of ambiguous Chinese pronouns, so that stronger conclusions on the interplay between structural and semantic factors could be drawn. Moreover, in view of the lack of statistical significance with the by-item analyses in Experiment 2, the 
Table 2

Mean Character Reading Times

(in Milliseconds; With Standard Deviations) in Experiment 2

\begin{tabular}{|c|c|c|c|c|c|c|c|c|}
\hline \multirow[b]{4}{*}{ Region } & \multicolumn{8}{|c|}{ Passage Type } \\
\hline & \multicolumn{4}{|c|}{ First Antecedent } & \multicolumn{4}{|c|}{ Second Antecedent } \\
\hline & \multicolumn{2}{|c|}{ Agent } & \multicolumn{2}{|c|}{ Patient } & \multicolumn{2}{|c|}{ Agent } & \multicolumn{2}{|c|}{ Patient } \\
\hline & $M$ & $S D$ & $M$ & $S D$ & $M$ & $S D$ & $M$ & $S D$ \\
\hline Ambiguity & 369.7 & 114.2 & 378.8 & 103.3 & 378.6 & 86.7 & 371.6 & 90.3 \\
\hline Disambiguation & 339.8 & 93.4 & 368.5 & 119.0 & 415.5 & 182.0 & 412.1 & 168.6 \\
\hline Final & 320.9 & 94.0 & 317.1 & 98.9 & 323.8 & 101.0 & 326.5 & 79.1 \\
\hline
\end{tabular}

number of stimulus items was increased in Experiment 3 in order to provide a more sensitive test.

\section{EXPERIMENT 3}

In Experiment 3, we examined whether semantically biasing information presented before the critical pronoun would override antecedent order as a factor for resolving Chinese pronouns. One way to introduce explicit semantic bias was to modify the critical pronoun with a preposed modifier phrase that described a characteristic belonging to one but not the other antecedent. Unlike English, the Chinese language permits the modification of pronouns by preposing modifier phrases, and the resultant structure is common in everyday use. In the present experiment, the preposed phrase (i.e., the context) and the disambiguating information introduced later always agreed on the identity of the pronoun. Examples of these modified test passages are presented in Figure 3.

If biasing semantic context does affect the initial interpretation of ambiguous pronouns and override the effect of antecedent order, the slowing in reading owing to antecedent order observed in the two previous experiments would be present only when neutral modifier phrases (describing a characteristic that applied equally well to the two antecedents) were preposed. Biasing modifiers, on the other hand, would eliminate the antecedent order effect.

\footnotetext{
Method

Participants. The participants were 48 undergraduates at the Chinese University of Hong Kong. None of them had participated in the preceding experiments. They were native Chinese speakers/ readers participating in the experiment for course credit.

Design and Materials. In addition to the 20 passage frames used in the previous experiment, 28 new frames were constructed, making a total of 48 frames in Experiment 3 . The new frames were designed in the same way as the old ones. A three-character modifier phrase was inserted into each of the 48 passages immediately before the critical pronoun. In half of the passages, the preposed modifier phrase semantically biased interpretation of the pronoun toward one particular antecedent by describing a characteristic that only applied to that antecedent. The other half of the passages contained preposed modifiers that were semantically neutral (i.e., they described a characteristic that applied to both antecedents). The antecedent noun phrases consisted of both common nouns $(31.3 \%)$ and proper names $(68.7 \%)$.
}

Apparatus and Procedure. The apparatus and procedure were the same as those in Experiment 2.

\section{Results and Discussion}

Effectiveness of manipulation. A second version was developed for each of the 48 passage frames in such a way that the positions of the antecedents were reversed in the new version. Both the context and the disambiguating phrase were removed from these passages, which were then presented to undergraduate judges at the Chinese University of Hong Kong. Ten independent judges decided on the identity of the pronoun for each version. Results showed that $67.1 \%$ and $61.4 \%$ of the responses were first-antecedent assignment decisions for the original and the reversed versions, respectively. These percentages indicated the ambiguity of the pronoun when neither the modifier nor the disambiguating phrase was available.

Comprehension accuracy rates. Overall, about $98 \%$ and $89 \%$ of the comprehension questions were answered correctly for the filler and the target passages, respectively. The majority of the passages were properly read and comprehended. The percentages of correct answers in the various experimental conditions are shown in Table 3. A within-subjects ANOVA revealed a significant main effect of antecedent order $\left[F(1,47)=5.29, M S_{\mathrm{e}}=124.4, p<\right.$ $.05]$, indicating that comprehension accuracies were particularly high when the first antecedent was disambiguated as the pronoun coreferent. All the other effects failed to reach significance.

Character reading times. Only data from properly understood passages (with correctly answered comprehension questions) were analyzed. Mean character reading times were derived for the ambiguity, disambiguation, and final regions. Mean character reading times were calculated by dividing the total reading time for each region by the number of characters in that region. They are presented in Table 3.

As in Experiment 2, only reading times in the disambiguation region were analyzed. By-subject $\left(t_{1}\right)$ and byitem $\left(t_{2}\right) t$ tests were performed in both the biased and the neutral context conditions to examine the effects of antecedent order. With a preposed biasing context, the order of antecedents did not affect reading times $\left[t_{1}<1 ; t_{2}(47)=\right.$ $1.40, p=.17]$. With a neutral context, however, the effect 


\section{English Translation of a Sample Sentence Frame Used in Experiment 3:}

I unintentionally found a yellowish photograph from a photo album. In the photograph, my mother and my younger sister fell asleep on the sofa. (The benevolent/ The cuddly/

The wearing T-shirt) she snuggled up against my (mother/ sister), smiling sweetly; it looked so warm.

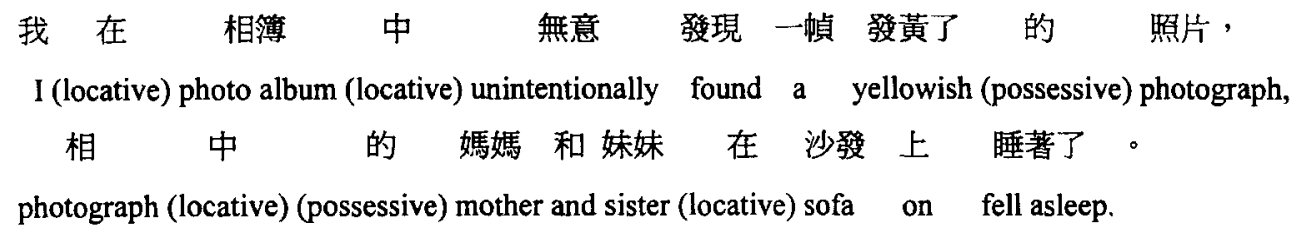

The Biased and First Antecedent (Bia-First) Version:

和荡可親的/她摟著 /(2) 姝妹

The benevolent (possessive)/(1)she snuggled /(2) sister

The Biased and Second Antecedent (Bia-Sec) Version:

小鳥依人的她摟著 / (2) 媽媽

The cuddly (possessive) /Dshe snuggled /(2) mother

The Neutral and First Antecedent (Neu-First) Version:

著短袖衫的她摟著妹妹

The wearing T-shirt (possessive)/(1) she snuggled /(2) sister

The Neutral and Second Antecedent (Neu-Sec) Version:

著短袖衫的她摟著 /(2) 媽媽

The wearing T-shirt (possessive)/(1) she snuggled /(2) mother

13), 咀莩泛起甜絲絲的微笑, 好一副溫整的樣子。/

/(3), mouth side showed sweet (possessive) smile, really a warm look./

(1)--ambiguity region
(2)--disambiguation region
(3)--final region

Figure 3. Sample passages used in Experiment 3 and their English translations.

of antecedent order again emerged $\left[t_{1}(47)=2.04, p<\right.$ $\left..05 ; t_{2}(47)=2.66, p<.05\right]$. As in the preceding experiment, reading in the disambiguation region slowed down when the information presented after the critical pronoun disambiguated it as the second antecedent, given that the preposed semantic context was consistent with both possible interpretations (i.e., a neutral context). Under this condition, the effect of antecedent order on reading times for the disambiguation region could not be explained by speed-accuracy tradeoff, since the slower reading times in the second-antecedent condition were accompanied by lower accuracy rates. Nevertheless, the effect of order on reading times was eliminated by the biasing semantic context, which was realized as a preposed modifier phrase that explicitly suggested the identity of the pronoun.

\section{GENERAL DISCUSSION}

Using natural Chinese passages, each containing a temporarily ambiguous pronoun preceded by two antecedents, 
Table 3

Mean Character Reading Times (in Milliseconds; With Standard Deviations) and Comprehension Accuracy Rates in Experiment 3

\begin{tabular}{|c|c|c|c|c|c|c|c|c|}
\hline \multirow[b]{4}{*}{ Region } & \multicolumn{8}{|c|}{ Passage Type } \\
\hline & \multicolumn{4}{|c|}{ Biased Context } & \multicolumn{4}{|c|}{ Neutral Context } \\
\hline & \multicolumn{2}{|c|}{ First Antecedent } & \multicolumn{2}{|c|}{ Second Antecedent } & \multicolumn{2}{|c|}{ First Antecedent } & \multicolumn{2}{|c|}{ Second Antecedent } \\
\hline & $M$ & $S D$ & $M$ & $S D$ & $M$ & $S D$ & $M$ & $S D$ \\
\hline Ambiguity & 255.6 & 78.1 & 265.0 & 79.3 & 265.3 & 75.6 & 261.5 & 69.0 \\
\hline Disambiguation & 261.9 & 86.0 & 265.5 & 83.1 & 262.9 & 86.6 & 282.5 & 90.9 \\
\hline Final & 224.8 & 87.3 & 226.7 & 80.5 & 229.7 & 62.7 & 237.9 & 85.5 \\
\hline $\begin{array}{l}\text { Comprehension } \\
\text { accuracy rates }(\%)\end{array}$ & 89.6 & & 85.8 & & 88.9 & & 85.2 & \\
\hline
\end{tabular}

the present study examined the effect of antecedent order on reading times in a subsequent text region that semantically disambiguated the pronoun. The effect of antecedent order was investigated when (1) the two antecedents carried the same theta role, (2) the antecedents carried different theta roles, and (3) a biasing semantic context was available before the ambiguous pronoun. Experiments 1 and 2 demonstrated that reading of the subsequent disambiguating information was slowed when it associated the pronoun with the second-appearing antecedent, as compared with the situation in which the information confined pronoun interpretation to the firstappearing antecedent. This antecedent order effect was independent of the theta roles of the antecedents. Experiment 3 showed that the effect of order was abolished by a preposed modifier phrase that semantically restricted pronoun interpretation. Taken together, these reading time data suggest a pronoun resolution preference that favors the first-appearing antecedent regardless of its theta role. This structurally based preference could, nonetheless, be overridden by semantic context. In general, the present findings are consistent with previous English results showing a subject assignment bias (Crawley et al., 1990; Gordon et al., 1993) and a notable effect of semantic context (Gordon \& Scearce, 1995).

Moreover, it is further argued that the well-documented subject assignment bias is based on the grammatical subject's appearing first, rather than on its role as performer of action in a normal English active construction. This could be considered an extension of the advantage of first mention, demonstrated in some previous research on English reading comprehension (e.g., Gernsbacher \& Hargreaves, 1988; Von Eckardt \& Potter, 1985). In a series of experiments, Gernsbacher and Hargreaves examined how recognition of sentential characters was affected by their various characteristics, as defined by sentence structure. Each test sentence described an activity involving two participants, and the critical dimension to be explored was their relative positions. The authors found that the first participant was recognized faster than the second participant when he/she was presented as a probe after the test sentence. Such an effect of first mention remained robust and independent even when the first participant was semantically the patient rather than the agent of the activity. The authors further showed that the advantage of first mention was not due to the first participant's initial position in the test sentence and that the effect persisted with joined-subject constructions in which the two participants were grammatically equivalent and linked by a conjunction to form a subject noun phrase. Gernsbacher (1989) showed that the first-mention effect was immediately suppressed by repeated proper name anaphors but was relatively preserved with pronoun anaphors. This result is consistent with the present finding that a temporarily ambiguous pronoun was more frequently associated with the first than with the second antecedent, because the first antecedent was the one that was more activated when reading came to the pronoun. An interpretation of the first-mention effect is offered by Gernsbacher (1990), who views it as evidence for her theory that the initial step to language comprehension requires the building of fundamental structures. The first participant temporally precedes the second one, and hence, it is preferentially attached to the more basic mental representation that is necessary for comprehension. In this sense, the initial mental structure that guides further comprehension is, to a significant extent, derived from the relative order of appearance of the critical sentential characters, regardless of their detailed semantic characteristics, such as theta role. This hypothetical mechanism of initial comprehension is entirely compatible with the major findings of the present study, which showed a first-antecedent advantage in pronoun resolution regardless of theta role. Therefore, the present findings also serve as supporting evidence for the structure proposed by Gernsbacher (1990).

The early semantic effect on the assignment of Chinese pronouns is in harmony with the context-dependent nature of the language. The present findings reinforce the argument of Miao (1981) and P. Li et al. (1992) for a central role of semantic cues in comprehending Chinese sentences by extending it to the particular case of pronoun resolution. Two new pieces of information are added to our current understanding. First, Chinese readers utilize semantic contexts (cues) not only in the comprehension of grammatically unnatural sentences (those used by Miao and $\mathrm{P}$. Li et al,), but also in the reading of perfectly natural passages. Second, semantic contexts not only influence processes that are themselves rather semantic in na- 
ture, such as the assignment of theta roles studied by Miao and P. Li et al., but also affect the structurally based process of how coreferents are identified for pronouns. These two generalizations tentatively point to the notion that context dependency in Chinese comprehension is a nonspecific phenomenon; it is generally observable across various processing domains.

\section{REFERENCES}

CHANG, F. R. (1980). Active memory processes in visual sentence comprehension: Clause effects and pronominal reference. Memory $\&$ Cognition, 8, 58-64.

CHEN, H.-C. (1992). Reading comprehension in Chinese: Implications from character reading times. In H.-C. Chen \& O. J. L. Tzeng (Eds.), Language processing in Chinese (pp. 175-205). Amsterdam: NorthHolland.

Chen, H.-C. (1996). Chinese reading and comprehension: A cognitive psychology perspective. In M. H. Bond (Ed.), The handbook of Chinese psychology (pp. 43-62). Hong Kong: Oxford University Press.

ChEN, H.-C. (1999). How do readers of Chinese process words during reading for comprehension? In J. Wang, A. W. Inhoff, \& H.-C. Chen (Eds.), Reading Chinese script: A cognitive analysis (pp. 257-278). Mahwah, NJ: Erlbaum.

ChEN, H.-C., \& HunG, W. W. (1995). Syntactic processes in Chinese reading: Some preliminary studies. Psychological Science, 18, 321-325.

Corbett, A. T., \& Chang, F. R. (1983). Pronoun disambiguation: Accessing potential antecedents. Memory \& Cognition, 11, 283-294.

Crawley, R. A., Stevenson, R. J., \& Kleinman, D. (1990). The use of heuristic strategies in the interpretation of pronouns. Journal of Psycholinguistic Research, 19, 245-264.

GERNSBACHER, M. A. (1989). Mechanisms that improve referential access. Cognition, 32, 99-156.

GernsBaChER, M. A. (1990). Language comprehension as structure building. Hillsdale, NJ: Erlbaum.

Gernsbacher, M. A., \& Hargreaves, D. (1988). Accessing sentence participants: The advantage of first mention. Journal of Memory \& Language, 27, 699-717.

Gordon, P. C., \& Chan, D. (1995), Pronouns, passives, and discourse coherence. Journal of Memory \& Language, 34, 216-231.

Gordon, P. C., Grosz, B. J., \& Gilliom, L. A. (1993). Pronouns, names, and the centering of attention in discourse. Cognitive Science, 17, 311-347.

Gordon, P. C., \& SCEARCE, K. A. (1995). Pronominalization and dis- course coherence, discourse structure and pronoun interpretation. Memory \& Cognition, 23, 313.323.

Greene, S. B., MCKoon, G., \& Ratcliff, R. (1992). Pronoun resolution and discourse models. Journal of Experimental Psychology: Learning, Memory, \& Cognition, 18, 266-283.

Haberlandt, K. F., \& Graesser, A. C. (1985). Component processes in text comprehension and some of their interactions. Journal of Experimental Psychology: General, 114, 357-374.

Just, M. A., \& CARPENTER, P. A. (1980). A theory of reading: From eye fixations to comprehension. Psychological Review, 87, 329-354.

Li, C., \& Thompson, S. (1981). Mandarin Chinese: A functional reference grammar. Berkeley: University of California Press.

Li, P., Bates, E., Liu, H., \& MacWhinner, B. (1992). Cues as functional constraints on sentence processing in Chinese. In H.-C. Chen \& O. J. L. Tzeng (Eds.), Language processing in Chinese (pp. $207-$ 234). Amsterdam: North-Holland.

MaCWHINNEY, B. (1987). Applying the competition model to bilingualism. Applied Psycholinguistics, 8, 315-327.

Matthews, A., \& ChoDorow, M. S. (1988). Pronoun resolution in twoclause sentences: Effects of ambiguity, antecedent location, and depth of embedding. Journal of Memory \& Language, 27, 245-260.

Mino, X.-C. (1981). Word order and semantic strategies in Chinese sentence comprehension. International Journal of Psycholinguistics, $\mathbf{8}$, 109-122.

Von Eckardt, B., \& Potter, M. C. (1985). Clauses and the semantic representation of words. Memory \& Cognition, 13, 371-376.

\section{NOTES}

1. Comprehension accuracy rates were higher for the filler than for the target passages in all three experiments. The difference was probably due to the fact that comprehension of fillers did not involve resolving ambiguous pronouns, whereas comprehension of target passages did. The relatively low accuracy rates associated with the target passages might thus reflect (1) that an extra processing load was needed for pronoun resolution or (2) that an ambiguous pronoun was not always linked by the reader to any particular entities.

2. For Experiments 1 and 2, contrasts of comprehension accuracy rates among the different experimental conditions were impossible, owing to data loss. Such contrasts were performed and reported only for Experiment 3.

(Manuscript received November 26, 1997; revision accepted for publication April 18, 1999.) 